\title{
Diseño de un cuestionario para conocer la satisfacción y percepción de los pacientes con el sistema de prescripción de receta electrónica
}

\author{
Amparo Bonilla Guijarro', Elena Bonilla Guijarro², Israel J. Thuissard Vasallo ${ }^{[}$, María Bailén Andrino ${ }^{4} \mathbb{E}$, \\ Asunción Hernando Jerez $z^{5}$
}

1. Licenciada en Farmacia. Farmacia comunitaria Amparo Bonilla, Madrid. 2. Graduada en Farmacia. Farmacia comunitaria Amparo Bonilla, Madrid. 3. Doctor en Biomedicina y Ciencias de la Salud. Universidad Europea de Madrid. 4. Doctora en Farmacia. Universidad Europea de Madrid. 5. Doctora en Medicina. Universidad Europea de Madrid.

\section{PALABRAS CLAVE}

Receta electrónica, cuestionario, fiabilidad, validez, farmacia comunitaria

\section{ABREVIATURAS}

CCI: coeficiente de correlación interclase

DE: desviación estándar

EVA: escala visual analógica

RIC: rango intercuartílico

\section{KEYWORDS}

Electronic prescription, questionnaire, reliability, validity, community pharmacy

\section{RESUMEN}

Objetivo: diseñar un cuestionario para conocer la satisfacción y percepciones de los pacientes con el sistema de prescripción de receta electrónica.

Métodos: el cuestionario se desarrolló en tres fases. Primera, búsqueda y selección de los atributos del cuestionario. Segunda, análisis del cuestionario por expertos mediante grupo focal multidisciplinar con farmacéuticos, médicos y enfermeros. Tercera, estudio piloto, observacional en 50 pacientes usuarios de receta electrónica durante al menos un año. Se valoró la consistencia interna del cuestionario, la fiabilidad intraobservador y la validez.

Resultados: de la primera y segunda fase resultó un cuestionario que incluye 5 atributos y 17 ítems. En el estudio psicométrico, el alfa de Cronbach fue de 0,77 (IC 95\%: 0,66-0,85), siendo el atributo de "satisfacción" el más valorado. La fiabilidad intraobservador tuvo un coeficiente de correlación de 0,96 (IC 95\%: 0,91-0,98). La validez de criterio quedó confirmada a través de la correlación de Spearman con una escala EVA. La validez convergente fue estadísticamente significativa $(p=0,04)$ para el nivel de estudios y no mostró relación con el número de tratamientos prescritos.

Conclusión: el cuestionario diseñado presenta adecuada fiabilidad y validez, y puede ser una herramienta útil para el trabajo en la farmacia comunitaria y para su uso en otras comunidades autónomas.

Design of a questionnaire to know the satisfaction and perception of the population with the electronic prescription system

Objective: Design of a questionnaire to determine the satisfaction and perceptions of patients with electronic prescription system.

Methods: The questionnaire was developed in three phases. First, search and selection of questionnaire attributes. Second, analysis of the questionnaire by experts with a focus group methodology with pharmacists, doctors and nurses. Third, observational pilot study with 50 patients, users of electronic prescription system for at least one year. The internal consistency of the questionnaire, intraobserver reliability and validity were assessed.

Results: The first and second phase resulted in a questionnaire that includes 5 attributes and 17 items. In the psychometric study, Croncach's alpha was 0.77 (CI95\%: 0.660.85 ), being the "satisfaction" attribute the one most valued. The intraobserver reliability had a correlation coefficient of 0.96 (CI95\%: 0.91-0.98). The validity of criterion was confirmed through Spearman's correlation with a VAS scale. Convergent validity was statistically significant $(\mathrm{p}=0.04)$ for the level of studies and did not show a relation with the number of prescribed treatments.

Conclusions: The designed questionnaire has adequate reliability and validity and can be a useful tool for work in community pharmacy and for use in other autonomous communities.
Recibido: $6 / 10 / 2020$

Aceptado: 18/3/2021

Disponible online: 19/4/2021
Financiación: ninguna.

Conflicto de intereses: ninguno.

Cite este artículo como: Bonilla A, Bonilla E, Thuissard IJ, Bailén M, Hernando A. Diseño de un cuestionario para conocer la satisfacción y percepción de los pacientes con el sistema de prescripción de receta electrónica. Farmacéuticos Comunitarios. 2021 Apr 19; 13 (2): 42-48. doi:10.33620/FC.2173-9218.(2021/Vol13).002.07

Correspondencia: farmacia.tribaldos@gmail.com

ISSN 1885-8619 @SEFAC (Sociedad Española de Farmacia Clínica, Familiar y Comunitaria). Todos los derechos reservados. 


\section{Introducción}

La receta electrónica se estableció en la Comunidad de Madrid en 2012, comenzando en los pueblos periféricos del sur, hasta su completa implantación en toda la región en diciembre de 2014 (1). Desde entonces, se ha extendido a las consultas hospitalarias, centros de especialidades, consultas de urgencias y se ha logrado la interoperabilidad con otras comunidades autónomas (2,3). Uno de los principales objetivos de la receta electrónica es que los pacientes con enfermedades crónicas no tengan que acudir a su centro de salud cada vez que necesitan medicación, reduciendo de esta forma la media mensual de visitas administrativas a los centros de salud hasta en un 60\% (4), así como centralizar en una sola tarjeta toda la medicación que recibe el paciente y mejorar la seguridad evitando duplicidades, interacciones, alergias y reduciendo hasta en un 60\% los errores en la prescripción al ser las recetas legibles, completas y en un formato estandarizado (5).

El conocimiento de la población del sistema de prescripción de receta electrónica ha ido en aumento, de un 56,2\% en 2012 (6) hasta un $88,7 \%$ en el 2016, cuando la consideraron bastante o muy útil en el 83,3\% de los casos (7). La tasa de adherencia a la prescripción electrónica también ha ido en aumento, desde el 49,6\% un año después de su completa implantación en toda la Comunidad de Madrid, hasta el 78,2\% en diciembre de 2018 (8). Sin embargo, a pesar de la elevada tasa de adherencia a la prescripción en receta electrónica de la población y aunque se han estudiado la percepción de los médicos a este sistema (9) o los motivos por los que los pacientes no retiran todos los medicamentos de su receta electrónica (10), nunca se ha estudiado el grado de satisfacción de los pacientes con este nuevo sistema.

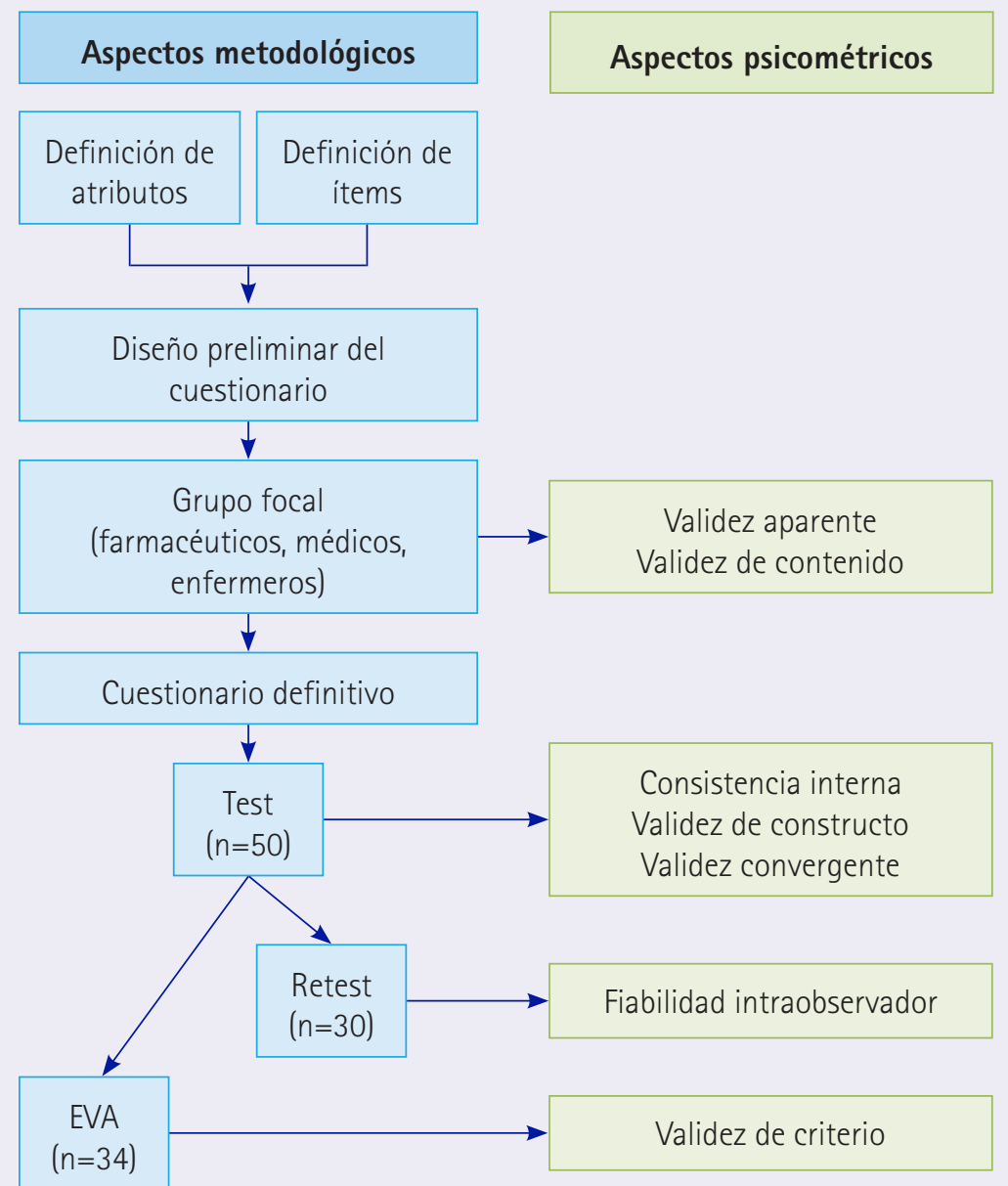

Figura 1 Metodología seguida en la validación del cuestionario y su relación con los aspectos psicométricos
Por ello, se consideró de interés el presente estudio, cuyo objetivo principal fue desarrollar un cuestionario que permita evaluar la satisfacción de los pacientes con la receta electrónica.

\section{Material y métodos}

El presente estudio forma parte del estudio "REACT: Receta Electrónica, Adherencia y Cumplimiento Terapéutico" en el que se analiza la adherencia de los pacientes crónicos a sus tratamientos mediante la receta electrónica y la satisfacción de los mismos con este sistema de prescripción a través del cuestionario cuyo diseño y validación presentamos en este artículo.

El diseño del cuestionario se llevó a cabo en tres fases: búsqueda y selección de los atributos del cuestionario, análisis del cuestionario por expertos mediante grupo focal y estudio piloto (figura 1).

\section{Búsqueda y selección de los atributos del cuestionario}

Se analizó el viaje del paciente desde que acude al médico por una consulta o a por una prescripción hasta que termina con la medicación dispensada en la farmacia comunitaria, analizando las interacciones que recibe con el sistema de receta electrónica. Para ello se trianguló la revisión de la bibliografía y la opinión de los expertos para determinar los atributos a introducir en el cuestionario. Se definieron los atributos como las diferentes cualidades o propiedades del sistema de receta electrónica que deben ser evaluados por su repercusión directa sobre el paciente. Finalmente, se incluyeron los siguientes atributos en el cuestionario:

\section{Satisfacción}

Es la medida en que el paciente valora todo el proceso de obtención de su medicación a través del sistema de receta electrónica de forma general.

\section{Acceso a los tratamientos}

Evalúa el grado en que el paciente ha podido retirar su medicación en la farmacia comunitaria siempre que lo ha necesitado, atendiendo al criterio de la Organización Mundial de la Salud sobre acceso; es decir, facultad de estar disponibles en la farmacia, lo que implica no solo que el medicamento está disponible, sino que 
lo está de forma sostenible y continua, de acuerdo a unos estándares de calidad establecidos y que, además, el paciente dispone de información para un uso racional del medicamento (11).

\section{Comprensión}

Es el atributo del cuestionario que hace referencia al sistema de plazos de 28 días del sistema. Los tratamientos crónicos en el sistema de receta electrónica están disponibles en periodos de 28 días y se van renovando de acuerdo con esos periodos. El atributo "comprensión" mide el grado en que el paciente entiende este sistema de plazos y su capacidad para interpretarlo.

\section{Gasto}

Mide la percepción del paciente sobre su aportación económica a sus tratamientos mediante el copago farmacéutico en comparación con el antiguo sistema de receta de papel (12).

Otros ámbitos de la receta

electrónica: interoperabilidad,

hospitales y urgencias

El atributo de interoperabilidad evalúa el grado en que el paciente ha podido retirar su medicación en otras comunidades autónomas en los últimos meses. Asimismo, en este atributo se evalúa la experiencia de adquisición de medicamentos después de una prescripción en un centro hospitalario por una visita programada o de urgencias (13).

Una vez definidos los atributos se redactaron las preguntas que habría que hacer a los pacientes en relación con estos y se optó por una escala Likert para evaluar las respuestas. Al finalizar esta fase, quedó definido un cuestionario con 14 ítems.

\section{Análisis por expertos de la} validez aparente y de contenido mediante grupo focal

Se utilizó la metodología de grupo focal para discutir la versión inicial del cuestionario. Participaron en el grupo diferentes profesionales sanitarios: dos farmacéuticos, dos médicos $\mathrm{y}$ dos enfermeros. Estos valoraron si en el cuestionario inicial estaban incluidos todos los items relevantes y si la redacción de los mismos era la adecuada para medir lo que se proponían.

\section{Estudio piloto}

Finalmente, para la validación del cuestionario se realizó un estudio piloto, observacional, de corte transversal y no intervencionista en una farmacia comunitaria de la Comunidad Autónoma de Madrid. En el estudio participaron 51 pacientes, si bien para el análisis de datos sociodemográficos solo se pudieron incluir 50 por tener un paciente datos inconsistentes. Los criterios de inclusión eran: ser mayor de edad, usuario del sistema de receta electrónica $\mathrm{y}$ con medicación crónica prescrita durante al menos un año de duración. Además, los pacientes no habían de padecer deterioro funcional o cognitivo, ni estar tratados con fármacos del grupo N06D (sistema nervioso, psicoanalépticos, anti-demencia: donepezilo, galantamina, memantina, rivastigmina y ginkgo). Los pacientes debían firmar voluntariamente el consentimiento informado y el documento garante de la ley de protección de datos.

El estudio se llevó a cabo de acuerdo con las guías de buena práctica clínica de la Declaración de Helsinki y fue aprobado por el Comité Ético de Investigación Clínica del Hospital Universitario Puerta de Hierro Majadahonda (código de protocolo ABGENA-2019-01), en la Comunidad Autónoma de Madrid.

Se reclutaron los cinco primeros pacientes de la mañana y los cinco primeros pacientes de la tarde. En la primera visita se recogieron los datos sociodemográficos de los pacientes, información sobre el número de tratamientos prescritos y las respuestas del cuestionario. Dos meses más tarde se pidió de nuevo la contestación del cuestionario a treinta de los pacientes iniciales para confirmar la estabilidad del cuestionario. Además, se utilizó una escala analógica visual (EVA) en la que los pacientes marcaban su satisfacción general con el sistema de receta electrónica.

\section{Análisis estadístico}

Las variables cuantitativas recogidas en el estudio se describen como media \pm desviación estándar (DE) si siguen una distribución normal o mediana [rango intercuartílico, RIC] en caso contrario. Se utiliza la prueba de Kolmogorov-Smirnov para conocer el comportamiento paramétrico de las variables. Para las variables cualitativas se utiliza la frecuencia absoluta (n) y la relativa (\%).

El análisis de la consistencia interna se realizó mediante el alfa de Cronbach. La fiabilidad test-retest se determinó a través del coeficiente de correlación intraclase (CCI).

Se analizó la validez convergente mediante la correlación de Spearman para el número de tratamientos prescritos y mediante el test ANOVA para el nivel de estudios.

Asimismo, se relacionaron los valores de satisfacción del cuestionario con la respuesta de los pacientes a la escala EVA cuando se les preguntaba por su satisfacción general para determinar la validez de criterio mediante la correlación de Spearman.

Se considera que un valor de significación por debajo del $5 \%(p<0,05)$ es estadísticamente significativo. Para el análisis estadístico se utilizó el programa SPSS v.23 (IBM Corporation, Armonk, NY, USA).

\section{Resultados}

\section{Descripción del cuestionario}

El primer borrador del cuestionario, resultado de la propuesta inicial de los investigadores, estaba formado por 14 ítems. En la discusión y el análisis con el grupo de expertos para garantizar la validez del contenido se consideró que estaban incluidos todos los atributos necesarios y que era preceptivo incluir tres nuevos ítems. El primero se relacionó con el acceso a los medicamentos que requieren visado para su dispensación. El segundo para valorar la comprensión de la receta electrónica y el hecho de que las prescripciones, aun siendo crónicas, no son indefinidas. Y el tercero para medir la necesidad de algunos pacientes de acudir al prescriptor sin cita para solucionar alguna incidencia relacionada con la receta electrónica. La versión final del cuestionario, con sus 17 items, queda reflejada en la tabla 1 .

\section{Estudio psicométrico}

\subsection{Datos sociodemográficos}

Para el primer test se reclutaron 50 pacientes. De ellos, 15 (30,0\%) eran hombres, con una media de edad de $69 \pm 10.4$ años. El resumen de las características sociodemográficas se muestra 
Tabla 1 Cuestionario de satisfacción con el sistema de receta electrónica. Relación de atributos e items

\begin{tabular}{|c|c|c|c|c|c|c|}
\hline Atributo & İtem & 1 & 2 & 3 & 4 & 5 \\
\hline \multirow[t]{2}{*}{ Satisfacción } & $\begin{array}{l}\text { Estoy satisfecho con el sistema de prescripción por } \\
\text { receta electrónica }\end{array}$ & & & & & \\
\hline & $\begin{array}{l}\text { Prefiero recoger mi medicación ahora, con el sistema } \\
\text { de receta electrónica, que antes con el sistema de re- } \\
\text { ceta de papel }\end{array}$ & & & & & \\
\hline \multirow{4}{*}{$\begin{array}{l}\text { Acceso a los } \\
\text { fármacos / } \\
\text { Tratamientos } \\
\text { desde Atención } \\
\text { Primaria }\end{array}$} & $\begin{array}{l}\text { Los medicamentos aparecen correctamente en la receta } \\
\text { electrónica cuando mi médico modifica o introduce un } \\
\text { tratamiento nuevo }\end{array}$ & & & & & \\
\hline & $\begin{array}{l}\text { Mi tarjeta sanitaria me permite recoger mi medicación } \\
\text { en la farmacia sin ningún problema }\end{array}$ & & & & & \\
\hline & $\begin{array}{l}\text { Frecuentemente, mi farmacéutico me dice que los me- } \\
\text { dicamentos que necesito no los manda el almacén, es- } \\
\text { tán desabastecidos y que vaya al médico a que me los } \\
\text { cambie }\end{array}$ & & & & & \\
\hline & $\begin{array}{l}\text { Nunca he tenido problema en recoger en la farmacia la } \\
\text { medicación que necesita visado }\end{array}$ & & & & & \\
\hline \multirow[t]{5}{*}{ Comprensión } & $\begin{array}{l}\text { Entiendo perfectamente que mis medicamentos van } \\
\text { apareciendo cada } 28 \text { dias en mi receta electrónica y que } \\
\text { antes de esos periodos no los puedo retirar }\end{array}$ & & & & & \\
\hline & $\begin{array}{l}\text { Entiendo que mi prescripción no es indefinida y debo } \\
\text { fijarme en la fecha de caducidad para renovarla }\end{array}$ & & & & & \\
\hline & $\begin{array}{l}\text { Mi médico me ha explicado cómo funciona el sistema } \\
\text { de receta electrónica y ha resuelto todas mis dudas }\end{array}$ & & & & & \\
\hline & $\begin{array}{l}\text { Mi enfermera me ha explicado cómo funciona el siste- } \\
\text { ma de receta electrónica y ha resuelto todas mis dudas }\end{array}$ & & & & & \\
\hline & $\begin{array}{l}\text { Mi farmacéutico me ha explicado cómo funciona el } \\
\text { sistema de receta electrónica y ha resuelto todas mis } \\
\text { dudas }\end{array}$ & & & & & \\
\hline \multirow[t]{2}{*}{ Gasto } & $\begin{array}{l}\text { Mi gasto en medicamentos se ha incrementado con el } \\
\text { sistema de receta electrónica }\end{array}$ & & & & & \\
\hline & $\begin{array}{l}\text { Independientemente de mi aportación económica, } \\
\text { el nuevo sistema de receta electrónica me compensa } \\
\text { personalmente }\end{array}$ & & & & & \\
\hline \multirow{4}{*}{$\begin{array}{l}\text { Otros } \\
\text { ámbitos de RE: } \\
\text { interoperabilidad, } \\
\text { hospitales y } \\
\text { urgencias }\end{array}$} & $\begin{array}{l}\text { He tenido problemas para utilizar la receta electrónica } \\
\text { fuera de la Comunidad de Madrid }\end{array}$ & & & & & \\
\hline & $\begin{array}{l}\text { He tenido problemas al recoger mi medicación con la } \\
\text { receta electrónica después de visitar urgencias. }\end{array}$ & & & & & \\
\hline & $\begin{array}{l}\text { He tenido problemas al recoger mi medicación con la } \\
\text { receta electrónica después de una consulta hospitalaria }\end{array}$ & & & & & \\
\hline & $\begin{array}{l}\text { He tenido que acudir a un centro de urgencia o a mi } \\
\text { médico sin cita para resolver algún problema relacio- } \\
\text { nado con la receta electrónica }\end{array}$ & & & & & \\
\hline
\end{tabular}

$1=$ Totalmente en desacuerdo. 2 = Desacuerdo. $3=\mathrm{Ni}$ de acuerdo ni en desacuerdo. $4=$ Acuerdo. $5=$ Totalmente de acuerdo. 
Tabla 2 Datos sociodemográficos de los participantes en el estudio

\begin{tabular}{|c|c|}
\hline Pacientes & $n=50$ \\
\hline Hombres & $15(30,0 \%)$ \\
\hline Edad (media) & 69 \\
\hline \multicolumn{2}{|l|}{ País de origen } \\
\hline España (\%) & $49(98,0 \%)$ \\
\hline Gambia & $1(2,0 \%)$ \\
\hline \multicolumn{2}{|c|}{ Nivel de estudios (\%) } \\
\hline Educación Primaria & $27(54,0 \%)$ \\
\hline FP / Bachiller & $16(32,0 \%)$ \\
\hline Universitario & $6(12,0 \%)$ \\
\hline Sin estudios & $1(2,0 \%)$ \\
\hline \multicolumn{2}{|l|}{ Situación laboral (\%) } \\
\hline Activo & $7(14,0 \%)$ \\
\hline Parado & $3(6,0 \%)$ \\
\hline Jubilado/Pensionista & $35(70,0 \%)$ \\
\hline Ama de casa & $4(8,0 \%)$ \\
\hline $\begin{array}{l}\text { En baja laboral / } \\
\text { Incapacidad }\end{array}$ & $1(2,0 \%)$ \\
\hline \multicolumn{2}{|c|}{ Situación familiar (\%) } \\
\hline Solo & $12(24,0 \%)$ \\
\hline Con pareja & $22(44,0 \%)$ \\
\hline Pareja con hijos & $10(20,0 \%)$ \\
\hline Con hijos & $5(20,0 \%)$ \\
\hline Con amigos & $1(2,0 \%)$ \\
\hline
\end{tabular}

Tabla 3 Consistencia interna: resultados del alfa de Cronbach

\begin{tabular}{|l|c|c|c|}
\hline & $\begin{array}{c}\text { Casos } \\
\text { evaluados } \\
\text { (n) }\end{array}$ & $\begin{array}{c}\text { Puntuación } \\
\text { (media } \pm \text { SD) }\end{array}$ & $\begin{array}{c}\text { Alfa de Cronbach } \\
\text { IC (95\%) }\end{array}$ \\
\hline Satisfacción & 51 & $86,0 \pm 22,9$ & $0,935(0,887-0,963)$ \\
\hline $\begin{array}{l}\text { Acceso a los fármacos/Tratamien- } \\
\text { tos desde Atención Primaria }\end{array}$ & 51 & $74,1 \pm 16,3$ & $0,551(0,310-0,723)$ \\
\hline Comprensión & 51 & $58,7 \pm 19,5$ & $0,581(0,367-0,739)$ \\
\hline Gasto & 51 & $81,6 \pm 17,0$ & $0,313(0,000-0,608)$ \\
\hline $\begin{array}{l}\text { Otros ámbitos de RE: interopera- } \\
\text { bilidad, hospitales y urgencias }\end{array}$ & 51 & $68,8 \pm 18,6$ & $0,520(0,262-0,704)$ \\
\hline Total & 51 & $70,6 \pm 12,8$ & $0,771(0,668-0,853)$ \\
\hline
\end{tabular}

en la tabla 2. Para el retest se reclutaron 30 pacientes y 34 para la respuesta a la escala EVA. Hubo 35 (70,0\%) pacientes pensionistas $\mathrm{y}$, con respecto al nivel de estudios, 27 (54,0\%) pacientes tenían como máximo nivel de estudios la educación primaria.

\subsection{Análisis psicométrico}

El esquema de la validación del cuestionario y de los aspectos psicométricos analizados puede verse en la figura 1. En relación con la consistencia interna, todos los atributos mostraron valores aceptables de alfa de Cronbach (tabla 3) con un valor total de 0,77 (IC 95\%: 0,66-0,85). Los atributos de satisfacción y otros ámbitos de receta electrónica (interoperabilidad, uso en hospitales y uso en urgencias) dieron los valores más altos: 0,93 y 0,52, respectivamente y destacando el atributo de gasto con el valor más bajo de alfa de Cronbach: 0,31 . El análisis de fiabilidad intraob- servador o test-retest arrojó un valor de CCI total de 0,96 (IC 95\%: 0,91$0,98)$, siendo la puntuación media en el test de $73,6 \pm 10,6$ y de $71,0 \pm 9,9$ en el retest (tabla 4).

Los resultados de la validez convergente muestran que al aumentar el nivel de estudios del paciente aumenta su puntuación en el cuestionario de forma estadísticamente significativa $(p=0,04)$. La puntuación media en el cuestionario para los pacientes con Educación Primaria es de 67,4; para los pacientes con Formación Profesional es de 73,7 y para los universitarios es de 78,2. El análisis ANOVA mostró que no existe relación entre el número de tratamientos prescritos $\mathrm{y}$ la puntuación en el cuestionario.

En la medida de la validez de criterio, la comparación con la puntación de la escala EVA de satisfacción con la receta electrónica obtuvo un coeficiente de correlación Rho de Spearman de 0,63 (IC 95\%: 0,48-0,78).

Tabla 4 Fiabilidad intraobservador o fiabilidad test retest

\begin{tabular}{|l|c|c|c|c|}
\hline & $\begin{array}{c}\text { Casos } \\
\text { evaluados } \\
\text { (n) }\end{array}$ & $\begin{array}{c}\text { Puntuación } \\
\text { test } \\
\text { (media } \pm \text { SD) }\end{array}$ & $\begin{array}{c}\text { Puntuación } \\
\text { retest } \\
\text { (media } \pm \text { SD) }\end{array}$ & $\begin{array}{c}\text { CCI } \\
\text { IC (95 } \%)\end{array}$ \\
\hline Satisfacción & 30 & $90,4 \pm 16,3$ & $86,7 \pm 15,0$ & $0,910(0,821-0,960)$ \\
\hline Acceso a los fármacos/Tratamientos desde Atención Primaria & 30 & $78,1 \pm 10,1$ & $74,6 \pm 9,7$ & $0,904(0,799-0,955)$ \\
\hline Comprensión & 30 & $60,8 \pm 20,4$ & $57,7 \pm 21,1$ & $0,965(0,927-0,984)$ \\
\hline Gasto & 30 & $83,3 \pm 16,8$ & $85,0 \pm 17,2$ & $0,984(0,966-0,992)$ \\
\hline Otros ámbitos de RE: interoperabilidad, hospitales y urgencias & 30 & $71,7 \pm 18,0$ & $69,2 \pm 18,7$ & $0,937(0,868-0,970)$ \\
\hline Total & 30 & $73,6 \pm 10,6$ & $71,0 \pm 9,9$ & $0,960(0,915-0,981)$ \\
\hline
\end{tabular}

CCl: coeficiente de correlación intraobservador. 


\section{Discusión}

Los cuestionarios, junto con la entrevista, son la técnica más utilizada en investigación sobre percepción, porque son menos costosos y permiten cuantificar y universalizar la recogida de información y estandarizar el proceso de la entrevista. Además, los cuestionarios permiten la comparabilidad de la información (14). Si bien la práctica clínica habitual no exige el uso de cuestionarios o escalas, estos suprimen el factor subjetivo del evaluador y la Organización Mundial de la Salud recomienda desarrollar y utilizar indicadores de la salud estandarizados y exige a la investigación multicéntrica internacional tener instrumentos validados en el ámbito de la salud para poder realizar estudios comparativos a nivel internacional (15). No obstante, los farmacéuticos refieren la falta de tiempo y la sobrecarga de trabajo como las principales barreras para investigar (16). Por ello, la factibilidad del cuestionario fue uno de los objetivos que nos propusimos al diseñarlo: que resultara sencillo, fácil de comprender por el paciente y que no demandara demasiado tiempo. Si bien no recogimos datos en este estudio piloto sobre el tiempo requerido para contestar el cuestionario o cuántos pacientes rechazaron participar en la investigación, no hubo ningún caso de pacientes que, una vez iniciado el cuestionario, lo dejaran a medias por su extensión, duración o falta de comprensión, lo que nos permite afirmar que conseguimos nuestro objetivo en lo que a factibilidad se refiere.

La mayoría de países de nuestro entorno dispone en la actualidad de sistemas de prescripción electrónicos más o menos avanzados y más o menos parecidos al nuestro y los beneficios económicos, sociales y sobre la salud de la receta electrónica están descritos en la literatura (17). En Suecia, el 85\% de los pacientes tiene una actitud positiva frente a la receta electrónica (18), en Estonia el 92\% de los pacientes que han usado la receta electrónica están satisfechos o muy satisfechos un año después de su implantación (19). Sin embargo, al ser la receta electrónica una herramienta de trabajo de farmacéuticos y médicos, la mayoría de los estudios sobre satisfacción y percepción se han centrado en los profesionales sanitarios y no han tenido en cuenta a los pacientes; aun cuando los pacientes nos pueden ofrecer una perspectiva única como principales beneficiarios de estos sistemas y es muy necesario ponerlos en el centro del sistema sanitario para mejorar el diseño y funcionalidad de los sistemas de información.

El grupo de expertos seleccionado para el grupo focal consideró que el cuestionario desarrollado abarcaba todas las dimensiones (atributos) con que la receta electrónica puede impactar en el paciente y enriqueció la visión de la receta electrónica más allá de la farmacia comunitaria, reforzando, una vez más, la necesidad de un trabajo multidisciplinar para el beneficio del paciente (20-22).

En relación con los diferentes aspectos de la fiabilidad del cuestionario, los valores de alfa de Cronbach observados pueden considerarse suficientes según algunos autores en las primeras fases de la investigación (23). Cabe destacar los valores especialmente altos en relación con la fiabilidad intraobservador o test-retest: a pesar de haber transcurrido más de dos meses entre los dos cuestionarios, los pacientes contestaron prácticamente igual en ambos casos, lo que da al cuestionario unos valores de fiabilidad entre buena y excelente.

El análisis de la validez convergente nos hizo ver que al aumentar el nivel de estudios del paciente aumenta también su puntuación en el cuestionario. Esto es coherente con las afirmaciones de algunos autores de que el analfabetismo se asocia con una menor adherencia $(24,25)$, lo que nos llevaría a desarreglos en la aparición de la medicación en la receta electrónica y a no poder retirar la medicación cuando fuera necesario. Por el contrario, no se encontró relación entre la satisfacción del paciente medida con nuestro cuestionario y el número de tratamientos prescritos, por lo que valdría la pena analizar qué otros factores se asocian con una mayor satisfacción del paciente con el fin de maximizar la mejora del sistema.

Por último, el análisis nos llevó a comparar la puntuación de nuestro cuestionario con la respuesta que daban los pacientes a la escala EVA de valoración de su satisfacción con la receta electrónica. Si bien existe una correlación entre ambas, aunque no sea muy elevada, demostrando que se cumple la validez de criterio, no podemos olvidar el hecho de que el cuestionario incluye en su valoración aspectos más técnicos o específicos que el paciente puede no valorar a simple vista en la EVA, tales como el acceso a medicamentos con visado, la interoperabilidad del sistema o aspectos económicos de la receta electrónica.

Este trabajo no está exento de algunas limitaciones. El cuestionario sólo se aplicó a población en una única farmacia comunitaria tanto en el test como en el retest. Al analizar los datos sociodemográficos de nuestra población se detecta una gran homogeneidad y dado que la consistencia interna de un instrumento puede variar según la población a la que se aplica (26), habrá que tenerlo en cuenta cuando el cuestionario se emplee en poblaciones con otras características. Asimismo, sólo se estudiaron pacientes adheridos al sistema de receta electrónica de la Comunidad de Madrid a pesar de que cada comunidad autónoma dispone de un sistema propio y diferente de dispensación por métodos electrónicos; sin embargo, los atributos analizados son inherentes a todos los sistemas, por lo que sería de interés para la comunidad científica el analizar las diferencias en satisfacción entre comunidades autónomas. Otra posible limitación es que el periodo de dos meses transcurrido entre el cuestionario inicial y el retest, según algunos autores (15), puede ser excesivo. En nuestro caso, la pandemia del COVID-19 no nos permitió ajustar más el periodo entre cuestionarios, pero los datos confirman uniformidad en los resultados.

En conclusión, puede afirmarse que el cuestionario diseñado para evaluar la satisfacción de los pacientes con el sistema de receta electrónica es válido y fiable y que puede ser una herramienta útil para el trabajo diario en la farmacia comunitaria y para su uso en otras comunidades autónomas.

\section{Agradecimientos}

A los participantes en el grupo de expertos durante la validación del cuestionario: los médicos José A. Andrés Llamas y M ${ }^{\text {a }}$ José Busto; las farmacéuticas Teresa Bueno e Isabel Casas; las enfermeras Mireia Santacreu y Delia Martínez. A la Dra. Cristina Andreu Vázquez, por su ayuda en el desarrollo de la metodología de la investigación. 


\section{Referencias bibliográficas}

1. Europa Press. Más de un millón de madrileños ya tienen acceso a la receta electrónica. 17 Febrero 2013. [Consultado 7/09/2020] Disponible en: https://www. europapress.es/madrid/noticia-mas-millon-madrilenos-ya-tienen-acceso-recetaelectronica-estara-todas-farmacias-2014-20130924150715.html

2. Ministerio de Sanidad Servicios Sociales e Igualdad. Sanidad pone en marcha la interoperabilidad de la receta electrónica entre Comunidades Autónomas. Nota de Prensa [Internet]. 2015 Jul 17. [Consultado 7/09/20]. Disponible en: https:// www.administracionelectronica. gob.es/pae_Home/pae_Actualidad/ pae_Noticias/Anio2015/Julio/Noticia-2015-07-07-interoperabilidad-receta-electronica-entre-CCAA.html

3. Servicio de Salud de la Comunidad de Madrid. Éxito en la implantación del Módulo Único de Prescripción en la Comunidad de Madrid. Nota de Prensa [Internet]. 2019 Oct 24 [Consultado 9/07/20] Disponible en: https://www.comunidad.madrid/ noticias/2019/10/24/exito-implantacion-modulo-unico-prescripcion-comunidad-madrid

4. Azagra Ledesma R, Aguyé Batista A. La prescripción electrónica en España. Aten Primaria [Internet]. 2005 [Consultado 7/9/2020]; 7/09/2035(9):457459. Disponible en: http://dx.doi.org/ 10.1157/13075477

5. Ferner RE. Computer aided prescribing leaves holes in the safety net. Br Med J. 2004;328(7449):1172-1173. doi:10.1136/bmj.328.7449.1172

6. Observatorio Nacional de Telecomunicaciones y de la Sociedad de la Información. La sociedad en red: Informe anual 2011 [Monografía en Internet]. Madrid: Editor Ministerio de Industria, Energía y Turismo, Secretaría de Estado de Telecomunicaciones y para la Sociedad de la Información; 2012. [Consultado 1/06/20] Disponible en: 17 https://www.ontsi.red.es/sites/ontsi/ files/ la_sociedad_en_red_2011_ed 2012.pdf

7. Vázquez Martínez R, Martínez López M. Los ciudadanos ante la e-sanidad. Opiniones y expectativas de los ciudadanos sobre el uso y aplicación de las TIC en el ámbito sanitario [Monografía en Internet]. Madrid: Editor Ministerio de Energía Industria y Turismo; 2016 [Consultado 1/07/20]. Disponible en: https://www.ontsi.red.es/sites/ontsi/ files/los_ciudadanos_ante_la_e-sanidad.pdf

8. Ministerio de Sanidad. Vicepresidencia Segunda del Gobierno. Ministerio de Derechos Sociales y Agenda 2030. Nivel de implantación de la Receta Electrónica en las Comunidades Autónomas. [Internet] Madrid, Ministerio de Sanidad, 2020. [Consultado 9/09/20] Disponible en: https://www. mscbs.gob.es/profesionales/recetaElectronicaSNS/home.htm

9. Villímar Rodríguez AI, Gangoso Fermosos AB, Calvo Pita C, Ariza Cardiel G. Percepción de los médicos de atención primaria sobre la receta electrónica en el servicio madrileño de salud. Rev Calid Asist. 2016;31(6):338-346. doi:10.1016/j.cali.2016.01.008

10. Prats Mas R, Botella Ripoll J, Dols Alonso D, Baixauli Fernández V, Climent Catalá MT. ¿Por qué los pacientes no retiran todos los medicamentos y productos sanitarios que les son prescritos por el médico? Farm Comunitarios. 2019;11(2):14-21. doi:10.5672/ FC.2173-9218.(2019/Vol11).002.03

11. Oscanoa T. Acceso y usabilidad de medicamentos: propuesta para una definición operacional. Rev Peru Med Exp Salud Publica. 2012; 29:119-145. doi:10.1590/S172646342012000100018

12. Jefatura del Estado. Real Decreto-ley $16 / 2012$, de 20 de abril, de medidas urgentes para garantizar la sostenibilidad del Sistema Nacional de Salud y mejorar la calidad y seguridad de sus prestaciones. Boletín Oficial del Estado, RDL 16/2012 2012. Disponible en: https://www.boe.es/buscar/act.php?i$\mathrm{d}=\mathrm{B} 0 \mathrm{E}-\mathrm{A}-2012-5403$

13. Servicio de Salud de la Comunidad de Madrid. Receta Electrónica. Ahora también en su hospital. [Internet]. Madrid, Consejería de Sanidad, Subdirección General de Farmacia y Productos Sanitarios. 2019. [Consultado 9/09/2020] Disponible en: http://www. madrid.org/bvirtual/BVCM020207.pdf

14. Martín Arribas MC. Diseño y validación de cuestionarios. Matronas Prof. 2004;5(17):23-9. Disponible en: https://www.federacion-matronas.org/ revista/wp-content/uploads/2018/01/ vol5n17pag23-29.pdf

15. Carvajal A, Centeno C, Watson R, Martínez M, Sanz Rubiales Á. ¿Cómo validar un instrumento de medida de la salud? An Sist Sanit Navar. 2011;34(1):63-72. doi:10.4321/S113766272011000100007
16. Awaisu A, Alsalimy N. Pharmacists' involvement in and attitudes toward pharmacy practice research: A systematic review of the literature. Res Soc Adm Pharm. 2015;11(6):725-48. doi:10.1016/j.sapharm. 2014.12.008

17. Deetjen U. European E-Prescriptions: Benefits and Success Factors [Internet]. 2016. [Consultado 9/09/2020]. Disponible en: https://www.politics.ox.ac. uk/materials/publications/15224/workingpaperno5ulrikedeetjen.pdf

18. Hammar T, Nyström S, Petersson G, Åstrand B, Rydberg T. Patients satisfied with e-prescribing in Sweden: A survey of a nationwide implementation. J Pharm Heal Serv Res. 2011;2(2):97-105. doi:10.1111/j.1759-8893.2011.00040.X

19. Parv L, Kruus P, Mõtte K, Ross P. An evaluation of e-prescribing at a national level. Informatics Heal Soc Care. 2014 Aug 12;41(1):78-95. doi:10.310 9/17538157.2014.948170

20. Hwang A, Gums TH, Gums JG. The benefits of physician-pharmacist collaboration. J Fam Pract. 2017;66(12):E1-E8. Disponible en: https://www.mdedge. com/familymedicine/article/152853/ diabetes/benefits-physician-pharmacist-collaboration

21. Smith SM, Carris NW, Dietrich E, Gums JG, Uribe L, Coffey CS, et al. Physician-pharmacist collaboration versus usual care for treatment-resistant hypertension. J Am Soc Hypertens. 2016;10(4):307-317. doi:10.1016/j.jash. 2016.01.010

22. Lagisetty P, Smith A, Antoku D, Winter S, Smith M, Jannausch M, et al. A physician-pharmacist collaborative care model to prevent opioid misuse. Am Heal Pharm. 2020;77(10):771780. doi:10.1093/ajhp/zxaa060

23. Nunnally J. Teoría Psicométrica. Editorial Trillas; 1967.

24. Sabaté E. Adherence to long-term therapies: evidence for action [Monografía en Internet]. WHO; 2003. Disponible en: https://apps.who.int/iris/bitstream/ handle/10665/42682/9241545992.pdf;jsessionid

25. Brown MT, Bussell J, Dutta S, Davis $\mathrm{K}$, Strong S, Mathew S. Medication Adherence: Truth and Consequences. Am J Med Sci. 2016 Apr 1;351(4):387-399. doi:10.1016/j.amjms.2016.01.010

26. Campo-Arias A, Oviedo HC. Propiedades psicométricas de una escala: La consistencia interna. Rev Salud Publica. 2008;10(5):831-839. doi:10.1590/ S0124-00642008000500015 